\title{
A Case Report of a Metastatic Adenocarcinoma of Lung with Dual Positivity for EGFR Mutation and ALK Fusion
}

\author{
Mangesh P Kamath ${ }^{1}$, Kadabur Nagendrappa Lokesh ${ }^{1 *}$, Govind Babu K ${ }^{1}$, Lakshmaiah KC ${ }^{1}$, Suresh Babu MC ${ }^{1}$ and Usha Amirtham ${ }^{2}$ \\ ${ }^{1}$ Department of Medical Oncology, Kidwai Memorial Institute of Oncology, Bangalore-29, India \\ ${ }^{2}$ Department of Pathology, Kidwai Memorial Institute of Oncology, Bangalore-29, India
}

"Corresponding author: Kadabur Nagendrappa Lokesh, MBBS, MD, DM, Assistant Professor, Department of Medical Oncology' Kidwai Memorial Institute of Oncology, Bangalore-29, India, Tel: 091-8971609070; E-mail: knloki@gmail.com

Received date: Aug 31, 2015, Accepted date: Oct 28, 2015, Publication date: Nov 05, 2015

Copyright: $\odot 2015$ Kamath MP, et al. This is an open-access article distributed under the terms of the Creative Commons Attribution License; which permits unrestricted use; distribution; and reproduction in any medium; provided the original author and source are credited.

\begin{abstract}
Non-small cell lung cancer ranks among the most lethal cancers worldwide. The rate of epidermal growth factor receptor (EGFR) mutations and echinoderm microtubuleassociated protein-like 4-anaplastic lymphoma kinase (EML4-ALK) gene fusion is the most common in younger age, non-smoking Asian adenocarcinoma lung cancer patients. EGFR mutations and ALK gene rearrangements are known to be mutually exclusive and as mutual causes of resistance to EGFR-tyrosine kinase inhibitors (TKIs) or ALK-TKIs. However, rarely such co-alterations do co-exist in some clinical cases. Here we report a 62 year old male, heavy smoker with cough, hemoptysis and fatigue. Histopathological examination of bronchoscopic guided biopsy showed adenocarcinoma histology. Staging evaluation, he was found to have stage IV disease on positron emission tomography-computed tomography scan. The biopsy blocks tested positive for both EGFR mutation and ALK fusion. Patient was initiated on tablet Gefitinib 250 mg once daily. To the best of our knowledge, there has been no report of dual EGFR mutation and ALK fusion positivity from India.
\end{abstract}

Keywords: Adenocarcinoma lung; Tyrosine kinase inhibitors; EGFR; Anaplastic lymphoma kinase; EGFR/ALK Co-alteration

\section{Introduction}

Lung cancer ranks among one of the most common and most lethal malignancies. Adenocarcinoma is the most commonly diagnosed histological subtype of non-small cell lung cancer (NSCLC) worldwide. Epidermal growth factor receptor (EGFR) mutations and echinoderm microtubule-associated protein-like 4-anaplastic lymphoma kinase (EML4-ALK) gene rearrangements are usually seen in adenocarcinoma histological subtype of lung. EGFR mutations and ALK rearrangements are mutually exclusive i.e ALK rearrangement is not seen in adenocarcinoma with EGFR mutation and vice-versa. Simultaneously, ALK rearrangements and EGFR mutations are known to be mutual causes of resistance to EGFR and ALK targeted therapies respectively.

To the best of our knowledge, there is no reported case of coexistent EGFR and ALK positive metastatic NSCLC from India. Here we report a case of a metastatic NSCLC found to be positive for both EGFR mutation and ALK rearrangement.

\section{Case Presentation}

A 62 year old male, working as a mechanic presented with cough with expectoration, intermittent hemoptysis and fatigue of 6 months duration. He was a heavy smoker for more than 40 years and had a history of alcohol abuse for more than 30 years. He had undergone coronary artery bypass grafting for ischemic heart disease 7 years ago after which he quit smoking and alcohol. On examination, he was moderately built and nourished with an Eastern co-operative oncology group (ECOG) performance status 1. On respiratory system examination, had a midline sternal healed scar, rhonchi in the right supraclavicular, infra-clavicular and axillary region. Other systems examination was unremarkable. Routine Haemogram and biochemistries were normal. Chest X-ray showed right upper zone non-homogenous opacity. Bronchoscopy showed a large right endobronchial ulcero-proliferative growth with right upper lobe collapse.

Bronchoscopic biopsy showed features conclusive of adenocarcinoma histology; the neoplastic cells were positive by Immunohistochemistry (IHC) staining for Cytokeratin 7(CK 7), Thyroid transcription factor 1 (TTF1) and Napsin A antibodies. A positron emission tomography- computed tomography (PET-CT) scan showed a perihilar right upper lobe mass lesion $(3.8 \times 3.5 \mathrm{~cm}$; standardized uptake value (SUV)max 24.3), metastatic mediastinal lymph nodes and multiple bilateral fluorodeoxyglucose (FDG) avid hypo-dense lesions in both the kidneys (Figures 1 and 2).

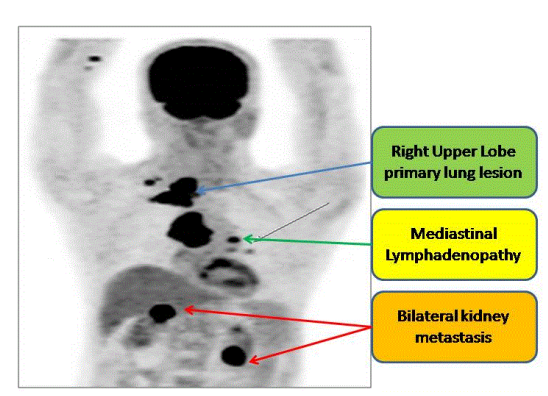

Figure 1: Baseline PET CT scan showing FDG avid right upper lobe lung lesions, mediastinal lymphdenopathy and bilateral kidney metastasis. 
Citation: Kamath MP, Lokesh KN, Babu KG, Lakshmaiah KC, Babu SMC, et al. (2015) A Case Report of a Metastatic Adenocarcinoma of Lung with Dual Positivity for EGFR Mutation and ALK Fusion. J Nucl Med Radiat Ther 6: 262. doi:10.4172/2155-9619.1000262

Page 2 of 3

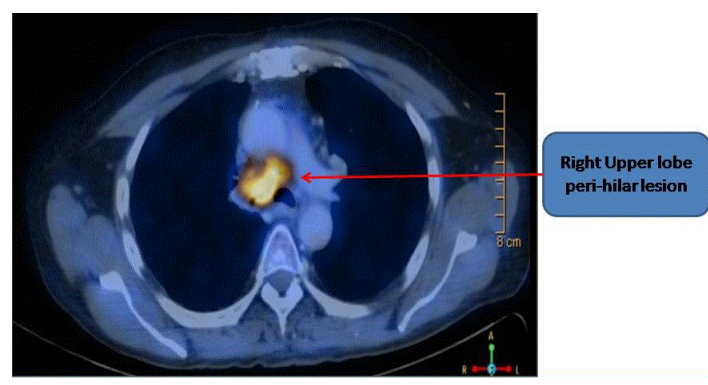

Figure 2: Baseline PETCT scan showing FDG avid right upper lobe perihilar lesions, mediastinal lymphdenopathy.

The paraffin blocks tested positive for EGFR mutation for G719X exon 18 (performed by amplification-refractory mutation system (ARMS) $^{\mathrm{mm}}$ and Scorpions Real time PCR method) (Figure 3) and EML4-ALK fusion (done by IHC for ALK D5F3 and confirmed by fluorescent in situ hybridization (FISH) analysis) (Figure 4). Patient was started on Tablet Geftinib $250 \mathrm{mg}$ once daily; he was initially better symptomatically for 1 month, but PET-CT scan after 3 months of starting Tablet Geftinib, showed progressive disease (Figure 5).

Patient was recommended but was not affording for ALK-TKI (Tablet Crizotinib) treatment. He was treated with 3 weekly chemotherapy with intravenous pemetrexed $500 \mathrm{mg} / \mathrm{m}^{2}$ and carboplatin area under curve (AUC) 5, both given on day 1 of each cycle. After 4 cycles of chemotherapy, patient was asymptomatic and reassessment with contrast enhanced computed tomography (CECT) scan showed a partial response. He was planned to continue on maintenance pemetrexed.

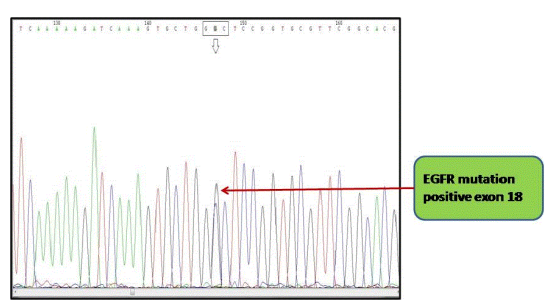

Figure 3: Tumour cells tested positive for EGFR mutation at exon 18 by the ARMS scorpion method.

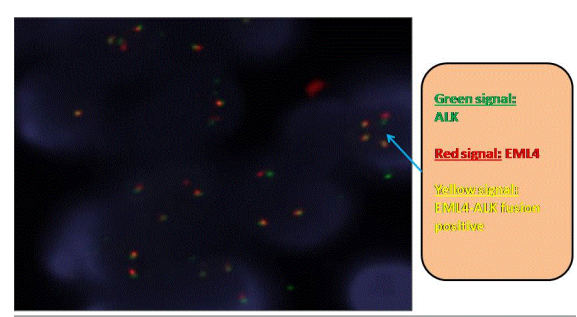

Figure 4: Tumour cells staining positive for EML4-ALK transloaction by fluorescent in situ hybridization (FISH) method.

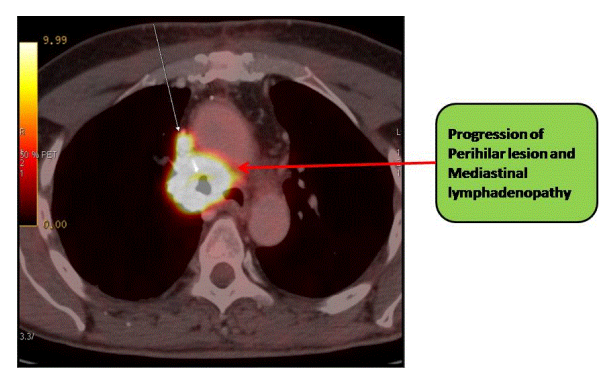

Figure 5: Re-assessment PETCT scan after 3 months of tablet Geftinib showing the progression of the FDG avid right upper lobe peri-hilar lesion and mediastinal lymphadenopathy.

\section{Discussion}

NSCLC with adenocarcinoma histology have been known to be genetically diverse. The understanding of its genetic diversity has led to the discovery of new molecular-targeted approaches. In particular, EGFR mutations and ALK gene rearrangements are the two most studied targets for the treatment of adenocarcinoma lung.

Similar to EGFR mutations, ALK rearrangements occur almost exclusively in young, Asian adenocarcinoma patients, who are either never or former smokers [1]. ALK rearrangements are not usually found in tumors with mutations in EGFR or Kirsten rat sarcoma viral oncogene homolog (K-RAS) mutated patients [2,3]. ALK rearrangements and EGFR mutations have been largely reported to be mutually exclusive [1] and as mutual causes of resistance to EGFR tyrosine kinase inhibitors (TKIs) and ALK TKI [4,5]. There is a possibility that similar to the prevalence of EGFR mutations, the coexistence of ALK rearrangement may be higher in East-Asian lung adenocarcinoma patients as compared to the Caucasians [6-8]. Recently, rare case reports have emerged noting the coexistence of EGFR mutation in EML4-ALK fusion positive patients [1,7-12]. However, the frequency of such co-alterations is yet to be fully described [9-11]. Yang et al. in a case series showed the frequency of dual EGFR and ALK co-alteration to be $\sim 1.3 \%$ and it was possibly higher in Chinese patients compared to the Caucasians [7]. Advanced molecular genetic technologies such as target next generation sequencing (NGS) with sensitivity to detect $<1 \%$ of EGFR mutation cells and FISH study for ALK rearrangements have enabled the detection of increasing numbers of cases with dual co-alteration [13]. It will be the key to detect many such new cases in the future and possibly alter the treatment strategies for such cases. Two hypotheses have been made to explain the existence of this concomitant alterationone of them being the differential genetic alteration occurring in the heterogenous tumor cell population and the other being the presence of a co-activating EGFR mutation in a homogenous cell population which has a pre-existing ALK gene rearrangement [12]. The most common co-existing EGFR mutations reported earlier included the Exon 19, L858R, L747P, and E868K [5]. In our patient, G719X exon 18 mutation was observed which is known to be sensitive to EGFR TKIs.

Knowing the possibility of Dual EGFR/ALK co-alteration may be of clinical relevance in terms of treatment strategies. First-line EGFRTKIs in TKI sensitive-EGFR mutant NSCLC have been shown to be superior to chemotherapy in terms of response rate, progression-free survival (PFS), quality of life and even have better survival rates 
[14,15]. Patients with ALK rearrangement benefit from the TKI Crizotinib [16]. However, for patients with concomitant EGFR mutations and ALK rearrangement, few reports are available regarding the clinical activity of EGFR-TKIs and ALK-TKIs [9-11]. Differential phosphorylation of EGFR or ALK which can be measured by IHC or the low burden of EGFR mutation in these cases might contribute to differences in sensitivity to EGFR-TKIs or Crizotinib. In a study by Won et al. concomitant EGFR and ALK gene alteration rates were noted in $0.3 \%$ patients [17]. When they studied the response rates in the dual positive cases with either of the TKI's, they found that the response rates with Gefitinib was disappointing while ALK inhibitors showed promising benefit in terms of response rate and progression free survival, similar to exclusive ALK rearrangement patients [16]. Our patient was treated with Geftinib; Crizotinib was not used in view of financial constraints. Similar to the study by Won et al. we also observed a poor response with Geftinib in our patient. This suggests that the dual positive patients are resistant to EGFR TKIs while retaining sensitivity to ALK inhibitors and that the clinical outcome of these patients with first line ALK inhibitors may be substantially better than with first line EGFR TKIs [17]. But the question of whether missing the concomitant EGFR mutation in ALK rearrangement positive patients has any impact on treatment outcome and prognosis is yet to be fully answered. The prevalence and clinical relevance of coalterations in these two driver genes require detailed investigation especially in the Asian countries which has high prevalence of EGFR.

\section{Conclusion}

EGFR/ALK co-alterations could define a specific subgroup that has diverse, although mostly favorable, responses to first-line TKI's. Testing of the relative phosphorylation levels of EGFR/ALK and EGFR mutations might help to guide the selection of TKIs in clinical practice. Molecular mechanisms underlying responsiveness and resistance to EGFR-TKIs and ALK-TKIs, and potential combination or sequential treatment require further investigation in this specific subgroup of coalterations. The true incidence, biology and treatment strategies of concomitant EGFR/ALK alteration need further evaluation in a large group of patients and for determining the effective management of such patients.

\section{References}

1. Shaw AT, Yeap BY, Mino-Kenudson M, Digumarthy SR, Costa DB, et al. (2009) Clinical features and outcome of patients with non-small-cell lung cancer who harbor EML4-ALK. J Clin Oncol 27: 4247-4253.

2. Inamura K, Takeuchi K, Togashi Y, Nomura K, Ninomiya H, et al. (2008) EML4-ALK fusion is linked to histological characteristics in a subset of lung cancers. J Thorac Oncol 3: 13-17.

3. Zhang X, Zhang S, Yang X, Yang J, Zhou Q, et al. (2010) Fusion of EML4 and ALK is associated with development of lung adenocarcinomas lacking EGFR and KRAS mutations and is correlated with ALK expression. Mol Cancer 9: 188.

4. Sasaki T, Koivunen J, Ogino A, Yanagita M, Nikiforow S, et al. (2011) A novel ALK secondary mutation and EGFR signaling cause resistance to ALK kinase inhibitors. Cancer Res 71: 6051-6060.

5. Doebele RC, Aisner DL, Le AT, Berge EM, Pilling AB, et al. (2012) Analysis of resistance mechanisms to ALK kinase inhibitors in ALK+ NSCLC patients. J Clin Oncol 30: 7504.

6. Sekine I, Yamamoto N, Nishio K, Saijo N (2008) Emerging ethnic differences in lung cancer therapy. Br J Cancer 99: 1757-1762.

7. Yang JJ, Zhang XC, Su J, Xu CR, Zhou Q, et al. (2014) Lung cancers with concomitant EGFR mutations and ALK rearrangements: diverse responses to EGFR-TKI and crizotinib in relation to diverse receptors phosphorylation. Clin Cancer Res 20: 1383-1392.

8. Lee JK, Kim TM, Koh Y, Lee SH, Kim DW, et al. (2012) Differential sensitivities to tyrosine kinase inhibitors in NSCLC harboring EGFR mutation and ALK translocation. Lung Cancer 77: 460-463.

9. Kuo YW, Wu SG, Ho CC, Shih JY (2010) Good response to gefitinib in lung adenocarcinoma harboring coexisting EML4-ALK fusion gene and EGFR mutation. J Thorac Oncol 5: 2039-2040.

10. Popat S, Vieira de Araújo A, Min T, Swansbury J, Dainton M, et al. (2011) Lung adenocarcinoma with concurrent exon 19 EGFR mutation and ALK rearrangement responding to erlotinib. J Thorac Oncol 6: 1962-1963.

11. Tiseo M, Gelsomino F, Boggiani D, Bortesi B, Bartolotti M, et al. (2011) EGFR and EML4-ALK gene mutations in NSCLC: a case report of erlotinib-resistant patient with both concomitant mutations. Lung Cancer 71: 241-243

12. Koivunen JP, Mermel C, Zejnullahu K, Murphy C, Lifshits E, et al. (2008) EML4-ALK fusion gene and efficacy of an ALK kinase inhibitor in lung cancer. Clin Cancer Res 14: 4275-4283.

13. Kim HS, Sung JS, Yang SJ, Kwon NJ, Jin L, et al. (2013) Predictive efficacy of low burden EGFR mutation detected by next-generation sequencing on response to EGFR tyrosine kinase inhibitors in non-small-cell lung carcinoma. PLoS One 8: e81975.

14. Zhou C, Wu YL, Chen G, Feng J, Liu XQ, et al. (2011) Erlotinib versus chemotherapy as first-line treatment for patients with advanced EGFR mutation-positive non-small-cell lung cancer (OPTIMAL, CTONG-0802): a multicentre, open-label, randomised, phase 3 study. Lancet Oncol 12: 735-742.

15. Yang JC, Schuler MH, Yamamoto N, O'Byrne KJ, Hirsh V, et al. (2012) LUX-Lung 3: A randomized, open-label, phase III study of afatinib versus pemetrexed and cisplatin as first-line treatment for patients with advanced adenocarcinoma of the lung harboring EGFR-activating mutations. J Clin Oncol 30: LBA7500.

16. Shaw AT, Kim DW, Nakagawa K, Seto T, Crinó L, et al. (2013) Crizotinib versus chemotherapy in advanced ALK-positive lung cancer. N Engl J Med 368: 2385-2394.

17. Won JK, Keam B, Koh J, Cho HJ, Jeon YK, et al. (2015) Concomitant ALK translocation and EGFR mutation in lung cancer: a comparison of direct sequencing and sensitive assays and the impact on responsiveness to tyrosine kinase inhibitor. Ann Oncol 26: 348-354. 